Fi ni te El ement Anal ysi s of Gal fenol Uni norph Vi br at i on Ener gy Harvester

\begin{tabular}{|l|l|}
\hline 著者 & $\begin{array}{l}\text { Rezaeeal am Behr ooz, Ueno Toshi yuki , Yanada } \\
\text { Sot oshi }\end{array}$ \\
\hline $\begin{array}{l}\text { j our nal or } \\
\text { publ i cat i on t i t l e }\end{array}$ & Nagnet i cs, I EEE Tr ansact i ons on \\
\hline vol une & 48 \\
\hline number & 11 \\
\hline page r ange & $3977-3980$ \\
\hline year & $2012-10-18$ \\
\hline URL & ht t p: //hdl . handl e. net /2297/36997 \\
\hline
\end{tabular}




\title{
Finite Element Analysis of Galfenol Unimorph Vibration Energy Harvester
}

\author{
Behrooz Rezaeealam $^{1,2}$, Toshiyuki Ueno ${ }^{1}$ and Sotoshi Yamada $^{1}$ \\ ${ }^{1}$ Division of Biological Measurement and Applications, Institute of Nature and Environmental Technology, Kanazawa University, \\ Kanazawa 920-1192 Japan \\ ${ }^{2}$ Department of Electrical Engineering, Lorestan University, Khorramabad, Iran
}

This paper develops a numerical model to examine the performance of the vibration energy harvester with one-rod (unimorph) of Iron-Gallium (Galfenol). The device's principle of operation is based on inverse magnetostrictive effect of the Galfenol rod. In order to take into consideration the anisotropy of the Galfenol, the Armstrong model is employed that is implemented into a static 3-D finite element model (FEM) of the energy harvester. The predicted results from the numerical model are compared to the measured ones.

Index Terms - Armstrong model, inverse magnetostrictive effect, energy harvester.

\section{INTRODUCTION}

$\mathrm{G}^{\mathrm{s}}$ alfenol is a promising transducer material that combines high magnetic susceptibility and desirable mechanical properties and therefore very suitable for harvesting vibration energy that involves bending stresses [1,2].

Previously, a bimorph vibration energy harvester has been developed [3] in which two rods of Galfenol are employed and capable of producing $10 \mathrm{~mW} / \mathrm{cm}^{3}$. The advantages of this energy harvester over the conventional ones, such as those using piezoelectric materials, are smaller size, higher efficiency and it also has high robustness and low electrical impedance.

In this paper, a unimorph-type of the device is proposed in order to consume less Galfenol and enhance the robustness of the device as one of the Galfenol rods is replaced by a stainless rod as shown in Fig. 1.

Static 3-D FEM is used to study the behavior of the energy harvester and the Multiphysics finite element package COMSOL allows the magnetostrictive strain tensor to be implemented directly using the actual properties of the materials involved within the system [4]. The Armstrong model is capable of predicting the multiaxial magnetoelastic behavior of magnetostrictive materials [5] and it could be incorporated in the finite element model of the whole system [6].

In this paper, the Armstrong model is developed for Galfenol $\left(\mathrm{Fe}_{81.6}\left(\mathrm{Ga}_{18.4}\right)\right.$ and the numerical model is employed in the design of the device and also to predict the performance of the energy harvester, and finally the calculated results are compared to the measured ones. The results show improvement in power density of the proposed energy harvester, that can be used to feed wireless sensors without the use of primary battery or can be placed inside the embedded structures wherein the appropriate ambient vibration exists.

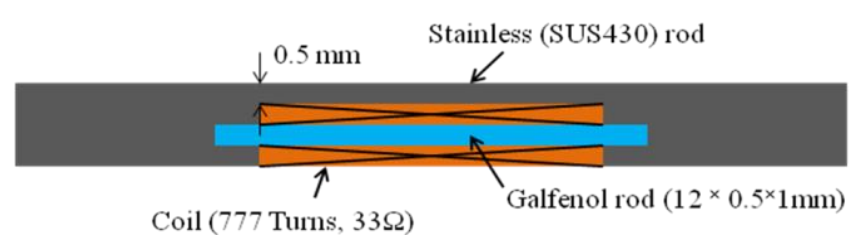

Fig. 1. The structure of the unimorph vibration energy harvester.

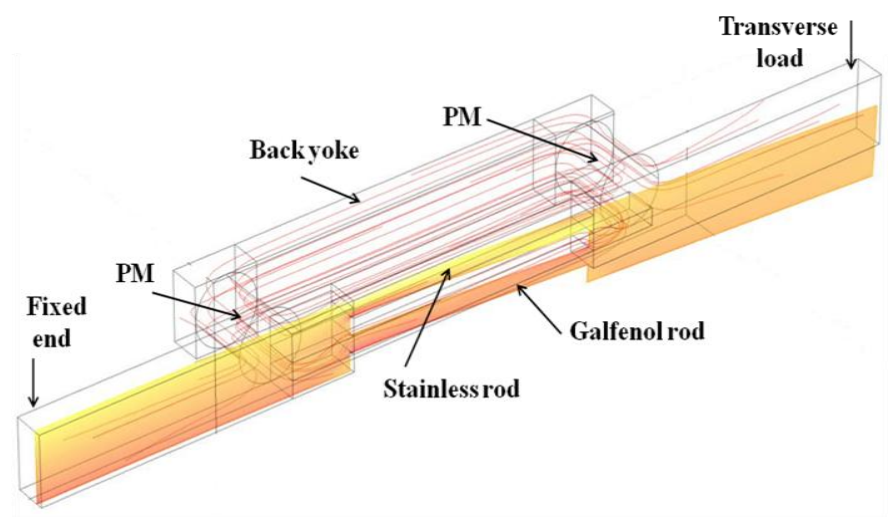

Fig. 2. The 3-D model of the Galfenol unimorph vibration energy harvester.

\section{GALFENOL UNimorph VibRATION ENERGy HARVESTER}

The Galfenol unimorph energy harvester consists of two parallel rods of which one is made of Galfenol $\left(\mathrm{Fe}_{81.6} \mathrm{Ga}_{18.4}\right.$, $0.5 \mathrm{~mm}$ by $1 \mathrm{~mm}$ area and $10 \mathrm{~mm}$ length, magnetically easy axis in longitudinal direction) and a coil of 777 turns is wound only on the Galfenol rod, as shown in Fig. 1. The other rod is made of stainless steel to improve the mechanical strength of the device as the Young's modulus of stainless is about 200 $\mathrm{GPa}$ while the one of Galfenol is around $70 \mathrm{GPa}$. Fig. 2 depicts the 3-D FEM view of the device in which one end is bonded to a fixture and the other end makes use of free vibration. Two pieces of Nd-B-Fe permanent magnets $(2 \mathrm{~mm}$ diameter and $2 \mathrm{~mm}$ length) are used to provide adequate bias flux for the rods and the attached back iron yokes close the magnetic circuit. 
The fundamental operating principle of the energy harvester is based on the inverse magnetostrictive effect that the magnetization changes with stress. when a transverse load is applied to the mover, one rod is compressed and the other one is stretched as shown in Fig. 2, leading to relative permeability change in the Galfenol rod, which causes the magnetic flux density to vary. Therefore, voltages are induced in the coils around Galfenol rod due to time-varying magnetic fields and the vibration energy is harvested.

\section{THE ARMSTRONG MODEL}

The Armstrong model is an energy-based model that the total energy corresponding to a particular orientation of magnetization for a given applied stress and magnetic field is evaluated as the sum of magneto-static $E_{\text {field }}$, the magnetocrystalline anisotropy $E_{a n}$, and the elastic energies $E_{\sigma}$ [5]. Using the total energy, as an ensemble average over all possible orientations of the magnetization vector is calculated to evaluate a macroscopic property of the material such as magnetization or magnetostriction. The local potential energy $W$ corresponding to the magnetization orienting in a direction $(\varphi, \theta)$ is given by:

$$
\begin{gathered}
W(\varphi, \theta)=E_{a n}\left(K_{1}, K_{2}, \varphi, \theta\right)+E_{\text {field }}\left(H, M_{s}, \varphi, \theta\right)+ \\
E_{\sigma}\left(\sigma, \lambda_{100}, \lambda_{111}, \varphi, \theta\right)
\end{gathered}
$$

The details of these energy terms are described in [5]. $K_{l}$ and $K_{2}$ are respectively -13.4 and $13.6 \mathrm{~kJ} / \mathrm{m}^{3}$ as the cubic and uniaxial anisotropies, $H$ and $\mu_{0} M_{s}=1.61 \mathrm{~T}$ are the magnetic field and saturation magnetization, also $\sigma, \lambda_{100}=170 \mathrm{ppm}$ and $\lambda_{111}=-4.7 \mathrm{ppm}$ denote the applied stress and magnetostriction parameters, respectively.

In this work, we will use the anhysteretic modeling technique which is deemed suitable for Galfenol as $\mathrm{Fe}-\mathrm{Ga}$ alloys exhibit negligible hysteresis. It is reasonable to assume that the orientation of the domains follows a Boltzmann distribution under the condition of non-interaction of domains as well as independence of the previous state (this assumption results in an anhysteretic model). The probability of the magnetization to be oriented along a direction $(\varphi, \theta)$ will therefore be:

$$
f(\varphi, \theta)=\frac{e^{-W(\varphi, \theta) / \omega}}{\int e^{-W(\varphi, \theta) / \omega} d \Omega}
$$

where $\omega$ is the potential distribution parameter and $d \Omega=\sin \theta d \theta d \varphi$. Then, the net magnetization in any direction is calculated using:

$$
M(\sigma, H)=\left\{\begin{array}{l}
M_{1} \\
M_{2} \\
M_{3}
\end{array}\right\}=\int M_{s}\left\{\begin{array}{l}
\sin (\theta) \cos (\varphi) \\
\sin (\theta) \sin (\varphi) \\
\cos (\theta)
\end{array}\right\} f(\theta, \varphi) d \Omega
$$

The expected value of magnetostriction $\lambda(\sigma, H)$ along any direction is similarly calculated by taking the ensemble average of $\lambda$ defined in terms of $\lambda_{100}, \lambda_{111}$ and direction of magnetization.

Before shaping to the rod, the Galfenol was stress-annealed

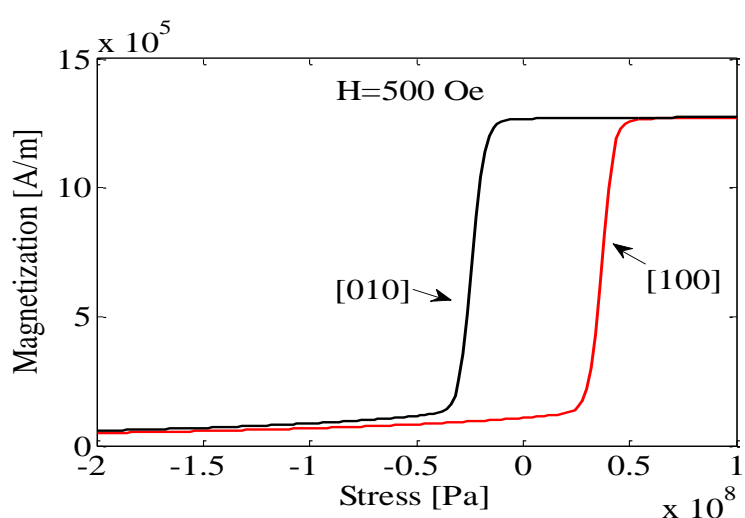

Fig. 3. Magnetization curves under uniaxial mechanical stress.

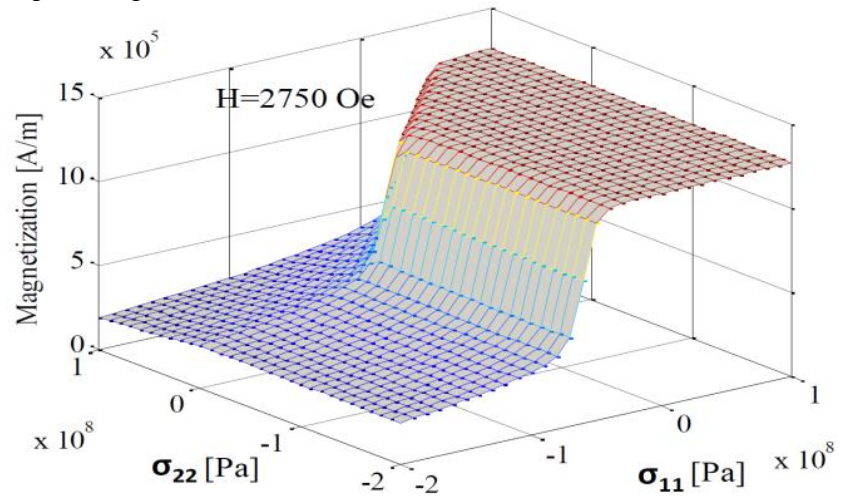

Fig. 4. Magnetization in direction [100] as a function of the principal stresses $\sigma_{11}$ and $\sigma_{22}$.

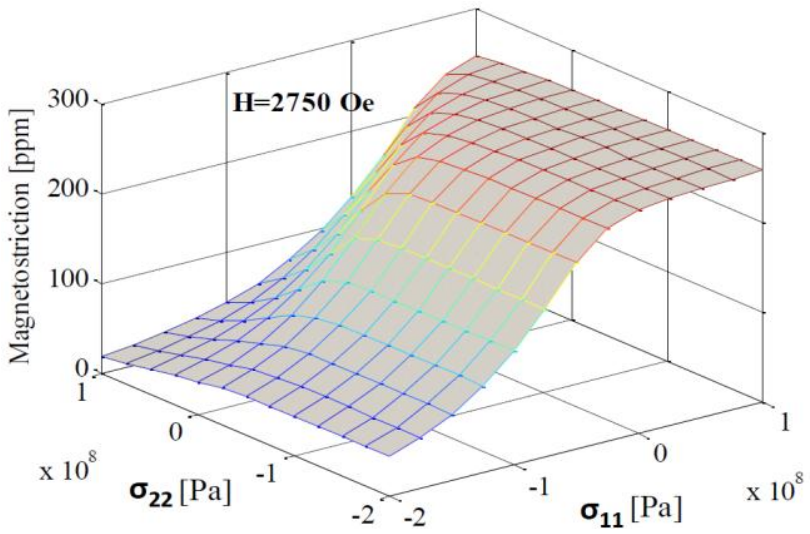

Fig. 5. Magnetostriction in direction [100] as a function of the principal stresses $\sigma_{11}$ and $\sigma_{22}$.

under compressive stress to equip built-in uniaxial anisotropy such that flux variation is occurred under tensile as well as compressive stresses.

For instance, at low bias fields such as 500 Oe applied along the easy axis [100], a tensile stress collinear to the bias field is needed to flip the magnetic moments toward the easy axis as shown in Fig. 3. While in case of applying the magnetic field in the perpendicular to the easy direction such as [010], the moments rotate easier toward the bias field as it happens in compressed stresses collinear to the bias field.

Figs. 4 and 5 illustrate the magnetization and the magnetostriction of Galfenol for an applied magnetic field of $2750 \mathrm{Oe}$ as a function of the applied bi-axial stress $\left(\sigma_{11}\right.$ and $\sigma_{22}$ being the principal stresses with $\sigma_{11}$ in the direction of the magnetic field). A compressive stress parallel to the magnetic field direction combined to a tensile stress perpendicular to the 
magnetic field direction results in a dramatic decrease of the material magnetization and magnetostriction. On the other hand a bitension mechanical loading hardly increases the magnetization and the magnetostriction.

The Armstrong model allows to define the magneto-elastic behavior of Galfenol with low computation cost and is then implemented into a finite element formulation, and will be applied to each element of the mesh.

\section{RESULTS}

By applying the bending moment, the energy harvester is deflected and the free end of device is displaced. Fig. 2 illustrates the 3-D model of the energy harvester and shows a slice cut through the Galfenol and stainless steel rods that demonstrates the spatial distribution of the principal stress $\sigma_{11}$ along the rods axis. In fact, the energy harvester resembles a cantilever as one of the rods is compressed and the other one is stretched. Fig. 6 shows that the magnetic flux density decreases inside the compressed Galfenol rod and it increases when the Galfenol rod is stretched. The corresponding alterations to the relative permeability inside the Galfenol rod are shown in Fig. 7, in which the tensile stress increases the relative permeability and the compression stress decreases the relative permeability. Galfenol has the advantage of larger variations in relative permeability from 20 to 300 due to both tensile and compression stresses in comparison to other magnetostrictive materials such as Terfenol.

The average flux densities component $B_{x \text {-ave }}$ inside the Galfenol and stainless rods along the axial direction are presented in Fig. 8 versus the displacement of the free end. The relative permeability of stainless steel is about 400 and is higher than the one of Galfenol and therefore the magnetic flux density in the stainless would be higher than the one in Galfenol. When the Galfenol rod is stretched, the tensile stress leads to an increase in the magnetization and thus the magnetic flux increases in Galfenol. Concurrently, as shown in Fig. 8, the magnetic flux decreases in stainless steel as a parallel path for the flow of the magnetic flux, because the magnetic reluctance of Galfenol reduces. Conversely, the compression stress causes a decrease in the magnetization, and therefore the magnetic flux density falls inside Galfenol.

The measurement setup is shown in Fig. 9, in which the energy harvester is connected to a shaker and a laser sensor is employed to measure the displacement caused by vibration. Fig. 10 shows the frequency response of the prototype and its resonant frequency of $1300 \mathrm{~Hz}$ is found. Another prototype of unimorph-type energy harvester in which the thickness of stainless rod reduced from 0.5 to $0.3 \mathrm{~mm}$, has been examined that the relevant resonant frequency is $1280 \mathrm{~Hz}$.

Both the above-mentioned prototypes have been vibrated at their resonant frequencies and the experimentally derived voltages and current of the coil wound around the Galfenol rod are shown in Figs. 11 and 12. The results show a large decrease of the output voltage and current by decreasing the thickness of the stainless rod.

The average flux density component $B_{x \text {-ave }}$ inside the Galfenol for the case of open-circuited coil has been

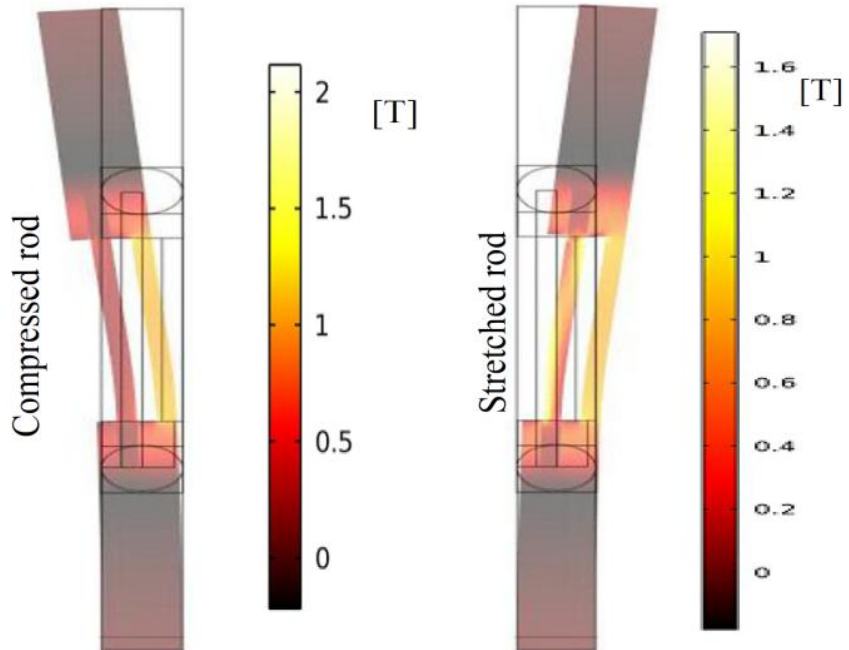

Fig. 6. Magnetic flux density distributions for two cases of compressed and stretched Galfenol rod.
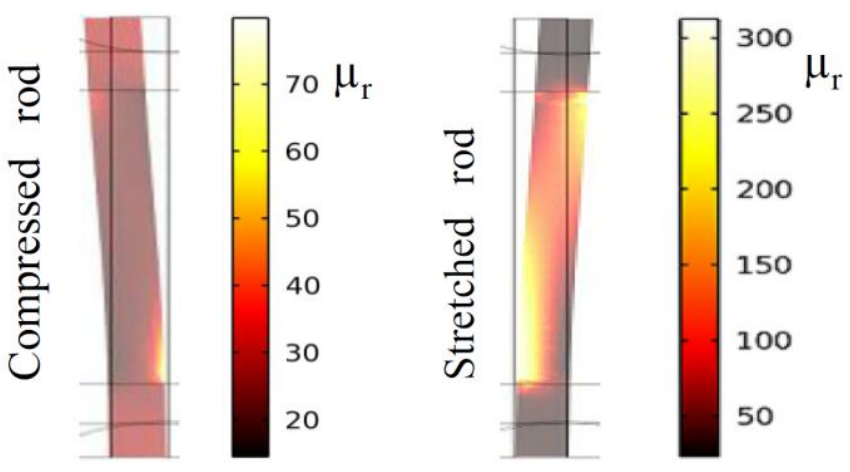

Fig. 7. Spatial variations of relative permeability inside the Galfenol rod.

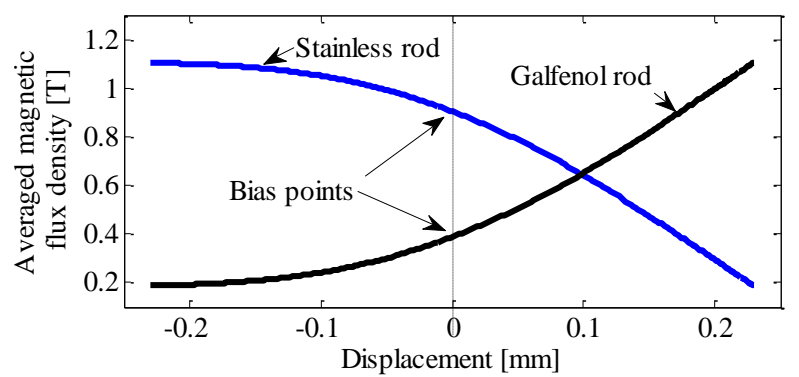

Fig. 8. Rod-averaged magnetic flux densities with stainless rod's thickness of $0.5 \mathrm{~mm}$.

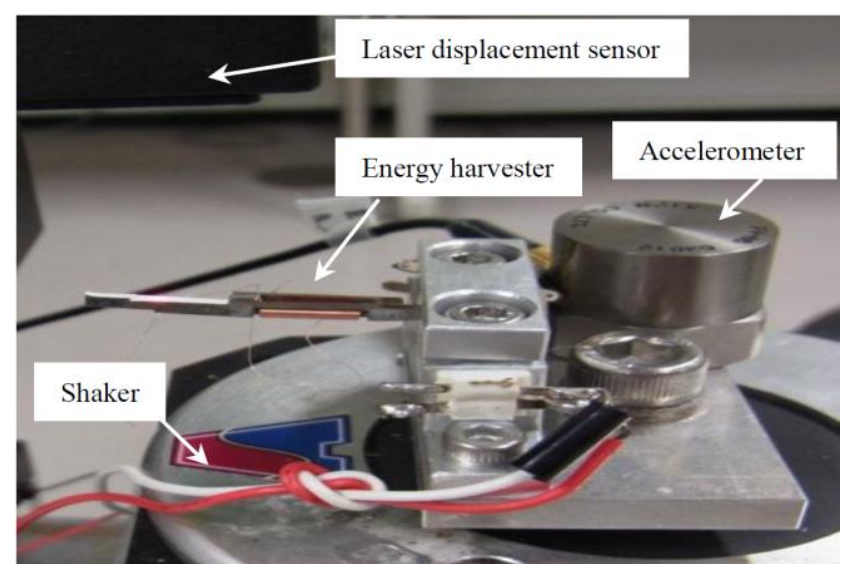

Fig. 9. Measurement setup 


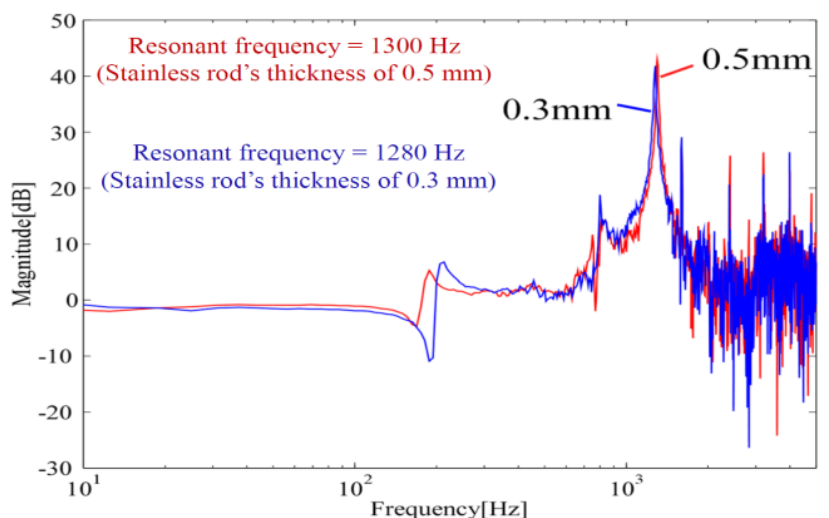

Fig. 10. Frequency response of both prototypes to determine their resonant frequency

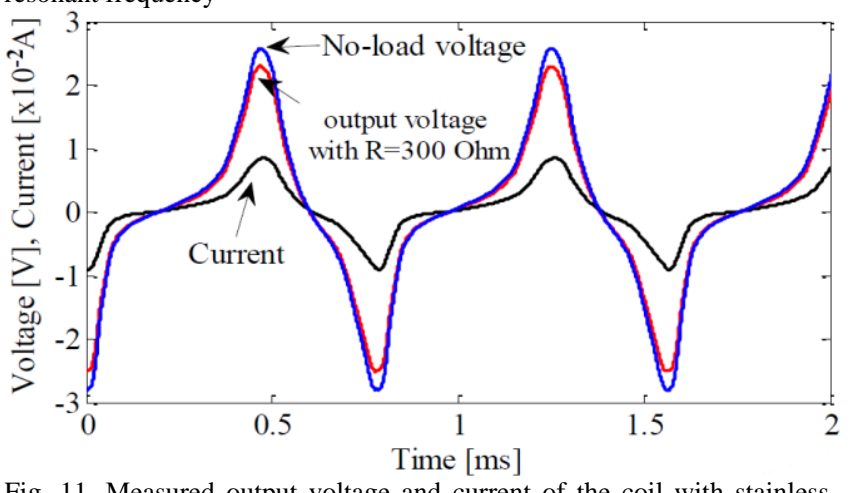

Fig. 11. Measured output voltage and current of the coil with stainless rod's thickness of $0.5 \mathrm{~mm}$.

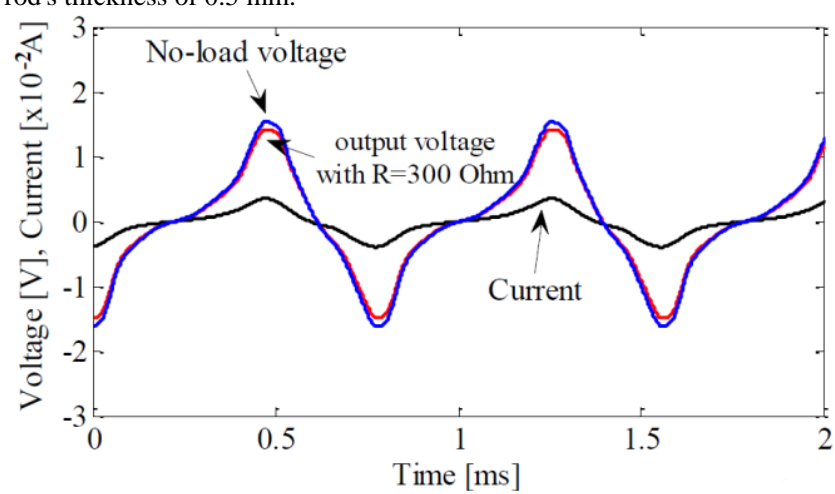

Fig. 12. Measured output voltage and current of the coil with stainless rod's thickness of $0.3 \mathrm{~mm}$.

determined using the measured open-circuit voltage induced in the coil around the Galfenol rod, which is in good agreement with the calculated one from the numerical model as shown in Figs. 13 and 14, including the corresponding displacement of the device's tip. For the prototype with stainless rod's thickness of $0.5 \mathrm{~mm}$, the variation of rod-averaged flux density is $0.87 \mathrm{~T}$ inside the Galfenol rod that shows the superiority of unimorph structure over the bimorph one, as the bimorph one provides $0.55 \mathrm{~T}$ inside each Galfenol rod [3].

The maximum deflection of $0.23 \mathrm{~mm}$ is measured for the case of stainless rod's thickness of $0.5 \mathrm{~mm}$, while it is reduced to $0.15 \mathrm{~mm}$ in the prototype with stainless rod's thickness of $0.3 \mathrm{~mm}$ at the resonant frequencies of the prototypes. This leads to a large decrease of magnetic flux variations inside the Galfenol which is seen in Figs. 13 and 14, because of lower stress applied to the Galfenol rod for the case with stainless rod's thickness of $0.3 \mathrm{~mm}$.

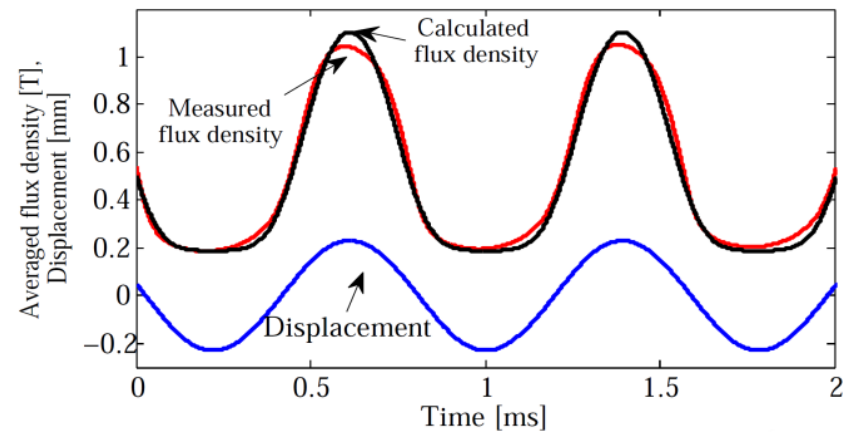

Fig. 13. Comparison between experimentally determined and model predicted rod-averaged magnetic flux density (stainless rod's thickness of $0.5 \mathrm{~mm})$

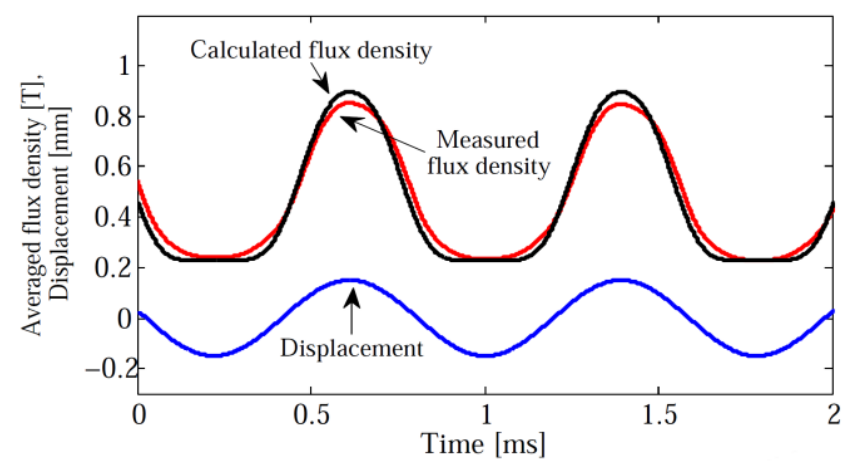

Fig. 14. Comparison between experimentally determined and model predicted rod-averaged magnetic flux density (stainless rod's thickness of $0.3 \mathrm{~mm})$.

\section{CONCLUSION}

In this paper, FEM coupled with 3-D Armstrong model has been employed to investigate the magneto-elastic behavior of Galfenol unimorph vibration energy harvester under multiaxial stresses as the active material of the device. the numerical model allows to understand how the relative configurations of stress and magnetic field modify the permeability. The numerical results agree with the measured ones and show the superiority of unimorph structure over the bimorph one due to less Galfenol consumption and larger variations in magnetic flux density that improves the effectiveness of the inspected device in voltage generation and energy harvesting applicable to wireless sensor networks as a lasting power supply.

\section{REFERENCES}

[1] J. B. Restorff, M. Wun-Fogle, A. E. Clark and K. B. Hathaway, "Induced magnetic anisotropy in stress-annealed Galfenol alloys," IEEE Trans. Magn., vol.42, no. 10, pp. 3087-3089, 2006.

[2] M. Wun-Fogle , J. B. Restorff and A. E. Clark, "Magnetomechanical coupling in stress-annealed Fe-Ga (Galfenol) alloys" IEEE Trans. Magn., vol. 42, no. 10, pp. 3120-3121, 2006.

[3] T. Ueno and S. Yamada, "Performance of Energy Harvester Using IronGallium Alloy in Free Vibration," IEEE Trans. Magn., vol. 47, no.10, pp. 2407-2409, 2011.

[4] COMSOL, MULTIPHYSICS @ (FEMLAB), [online]. http://www.comsol.com

[5] W. D. Armstrong, "A directional magnetization potential based model of magnetoelastic hysteresis", Journal of Applied Physics, vol. 91, pp. 2202-2210, 2002.

[6] C. Mudivarthi, S. Datta, J. Atulasimha and A. B. Flatau, "A bidirectionally coupled magnetoelastic model and its validation using a Galfenol unimorph sensor", Smart Materials and Structures, vol.17, pp.1-8, 2008 